\title{
Highly Configurable Low Cost Remote Laboratory with Integrated Support for Learning: Hardware Design
}

\author{
http://dx.doi.org/10.3991/ijoe.v9iS3.2527 \\ D. A. H. Samuelsen and O. H. Graven \\ Buskerud University College, Kongsberg, Norway
}

\begin{abstract}
This paper presents a flexible and low cost design specification for a remote laboratory platform for electronics laboratories. The system utilises a motherboard design and this paper presents both the design of the motherboard, various designs for add on cards and shows how to configure the setup. The design is focused on providing the user with a combination of easy setup and a large freedom in reconfiguration all provided as a low cost solution. The solution is designed to facilitate a natural flow in the creation of all forms of remote laboratories, from the prototyping level up to a permanent installation. This motherboard has connector for both configuration cards, programmable and static as well as for standardized add-on boards for all the configurable components in the laboratory circuit. Acquisition hardware can easily be changed both during development and after the creation of the lab.
\end{abstract}

Index Terms-Remote laboratory, hardware setup, reconfiguration.

\section{INTRODUCTION}

Laboratory exercises are an important part of engineering education, and should aid the theoretical understanding, as well as give hands-on experience for the students[1]. Remote laboratories are used extensively in universities all over the world and the number of publications in this area is large. A few can be found in [2-5], where the topic for all of them is within engineering education. This is typical as the design, implementation and maintenance of remote laboratory installations require a range of skills typically found among engineers. Maybe for this reason, remote laboratories have become very popular in engineering education. Another motivation can be found in the benefits they offer both to students as well as to the universities. For universities, the use of remote laboratories means reduced maintenance costs as the equipment can be protected from destructive use or simply because the wear and tear of the equipment is reduced. The cost of running laboratories for large groups of students is mostly eliminated, allowing the universities to route resources in other areas where students need extra follow-up. For students remote laboratories allows access to the laboratory $24 / 7$, at home or school at their choice. The drawbacks of using remote laboratories, like reduced contact with the students and lack of hands-on experience, are often overshadowed by the economic benefits. It is therefore reasonable to assume that remote laboratories will continue to be implemented at institutions around the world.
In general, remote laboratory installations can be divided into two main parts: the software frontend and the hardware backend. There is a large variety of software solutions available for the software frontend, and several tools tailored for this specific purpose are currently under development by large parties such as iLab[6], Lab2Go[7] and eLabs FEUP [8]. Much effort is put into the standardisation of the software, which is still under development.

In recent years it have emerged various ready-made solutions for the hardware backend. These are however very specialised, and offer little or no options for the user to implement laboratory exercises where the contents is adapted to the learning objectives of the individual modules of the university. This leads to these laboratories being mostly designed at the individual institutions, making it necessary for the institutions to spend a significant amount of resources on the development of the hardware backend. This is for many institutions not an option due to lack of resources or time, and the solution is often to buy off-the-shelf components that are built for other purposes, but . These however, offer a very low degree of flexibility for the institutions to develop laboratories tailored for the specific modules that the students follow. The authors therefore see a need for a remote laboratory platform that is both low-cost, easy to build and adaptable with regard to reconfiguration as the modules and hence, the design of the remote laboratory changes.

\section{SySTEM DESIGN}

\section{A. Requirements}

After deciding on an overall design specification and prior to the design process, a number of requirements were defined:

- The design should be modular in the sense that it should allow for easy reconfiguration by adding or removing more or less standardized components, as required by the laboratory setup.

- The platform should have a motherboarddaughterboard design. The motherboard must be able to accommodate the circuit boards for building the circuit under test. These are from now on referred to as component boards.

- The motherboard should have connectors for 14 component boards. Connectors should be according to a defined standard. The number 14 is a compromise between the number of add-on boards that 
can physically fit on one motherboard, and the number of add-on boards needed to create certain setups. With 14 add-on boards it is possible to set up a two-stage discrete amplifier, as well as several circuits involving operational amplifiers, such as active filters and oscillators.

- The motherboard should have a connector for an adapter to connect the acquisition module to the motherboard. A set of adapters must be designed for connecting one of the many different acquisition solutions to the motherboard. This is done in order make the platform easy adaptable to the different acquisition solution at the choice of the institutions.

- The component boards will constitute one or more of the components of the circuit to be tested in the laboratory. They should also confer to the interface description of the motherboard's connectors given in table 1 .

- The components of the experiment circuit that are implemented in the component boards should be configurable through the use of a simple data and control bus.

\section{B. Motherboard setup}

The motherboard is designed so that different component boards can be connected to each of the 14 component board connectors. The physical layout is shown in figure 1. Each component board constitutes one or more components with two or three connection points combined. Typically, a resistor will have two connection points, while a transistor or voltage divider will use three connection points. Devices with more connection points besides negative and positive power supply and ground, is not supported by this setup, meaning that operational amplifiers will be supported if their power supply pins are connected to the common positive and negative power supply or ground. Alternatively, a special component board can be designed and inserted into the analogue interconnect block described in the next paragraph, as this contains connections for all the other modules on the motherboard.

In order to configure the interconnection of the different components of the laboratory circuit, a separate configuration block is used. In figure 1 this is shown as the large connector on the left side of the board. Table 1 shows the pin assignment for the configuration block, and the number of pins used for each type of signal. The sum of pins used is 96, and a suitable choice for connector was found in the DIN-standard DIN-41612 where 96 pins are distributed as 32 pins in three rows. The connector was chosen due to its wide spread use in industry applications. In addition, this setup allows the user to make the interconnections either using wire wraps if long pins are mounted on the connector, or by the use of a printed circuit board for more permanent setups. This is further described in the next section.

Each of the three analogue connection pins of all the component board connectors is found in the configuration block; a total of 42 pins for the 14 boards. For the configuration of a laboratory experiment circuit, these need to be hardwired either by strapping or by a separate circuit board.
TABLE I.

PIN ASSIGNMENT FOR THE CONFIGURATION BLOCK

\begin{tabular}{|c|c|c|c|}
\hline \multirow{2}{*}{$\begin{array}{l}\text { Signal } \\
\text { type }\end{array}$} & \multicolumn{3}{|l|}{ Signals } \\
\hline & Purpose & $\begin{array}{l}\text { Short } \\
\text { name }\end{array}$ & $\begin{array}{c}\text { Number } \\
\text { of pins }\end{array}$ \\
\hline \multirow{4}{*}{ Power } & Power supply for the lab circuit & $\mathrm{V}+, \mathrm{V}-$ & 2 \\
\hline & Ground for lab circuit & AGND & 18 \\
\hline & Logic power supply & Vec & 1 \\
\hline & Ground for logic & DGND & 1 \\
\hline \multirow{3}{*}{ Analogue } & $\begin{array}{l}\begin{array}{l}\text { Analogue output from the } \\
\text { circuit }\end{array} \\
\end{array}$ & AOUT & 6 \\
\hline & Analogue input from the circuit & AIN & 16 \\
\hline & Connections of the circuit & $\mathrm{BxCy}$ & 42 \\
\hline \multirow{3}{*}{ Digital } & Data bus & $\begin{array}{c}\text { DATA } \\
x \\
\end{array}$ & 8 \\
\hline & Enable control signals & $\mathrm{ENx}$ & 2 \\
\hline & Total number of pins & & 96 \\
\hline
\end{tabular}

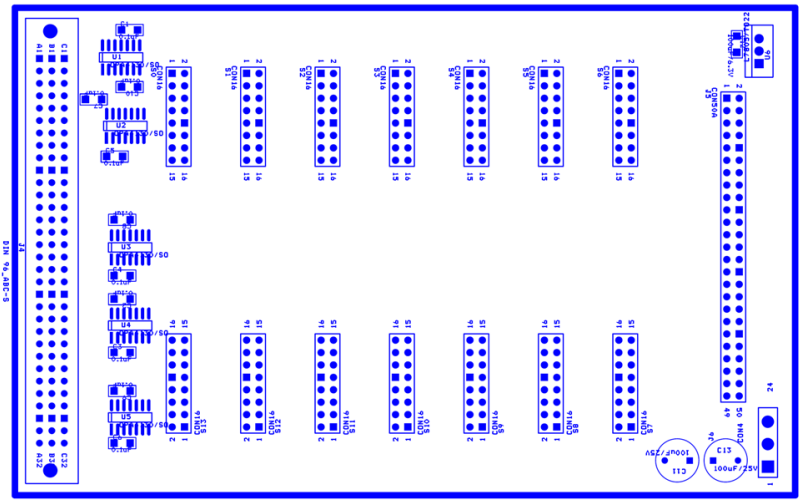

Figure 1. Board layout for the connectors on the motherboard.

Each of the three analogue connection pins of all the component board connectors is found in the configuration block; a total of 42 pins for the 14 boards. For the configuration of a laboratory experiment circuit, these need to be hardwired either by strapping or by a separate circuit board.

The justification for this arrangement is that the desired scheme for the development of remote laboratories can be divided into two phases: First, the prototyping phase where different setups are tested in order to verify their operations, and second the production phase where the best working prototype is being implemented as a more permanent setup. During the prototyping phase, the connections done in the configuration block are likely to be changed before the final layout is found. In this development process, strapping should be used until a stable layout is found. After a more or less extensive testing of the layout, a circuit board should be used to make the setup more robust against random errors due to temperature swing and mechanical failures. This should also be done in order to improve both the ability of the circuit to mimic a real circuit, as well as the quality of the measurements, as a tangle of long wires exhibit tendencies of crosstalk between the wires.

The configuration block is also given a secondary purpose as all the connections for two component boards are also fed to this block. This is done in order to facilitate the 
use of more complex circuit components, requiring more connections than the three connections available for the general component boards. This is briefly described in the first paragraph of this subsection. By placing this together with the configuration block, the limitation on the number of pins to be connected to each configurable component is removed as all connection to the other boards are available on this connector. In addition the data bus and two enable signals are tied to this connector, allowing for more complex configurations of components.

An alternative to the use of hardwired connections in the configuration block could be to use analogue multiplexers or switches. It is considered that the number of switches in such a switching matrix must be kept relatively low, as a large number of switching elements combined will exhibit a capacitance that would affect the overall properties of the laboratory circuit significantly. It can however be shown that a quite versatile setup for implementation of the vast majority of circuits required in an analogue electronics module can be done using a relatively small number of switches $(<20)$ in the configuration block, combined with the component boards. This must be seen in opposition to other suggested experiment setups requiring a stupendous amount of switching elements that is needed to cover all possible connections. If we only consider connections between neighbour pins in the configuration block of the setup presented in this paper this would give over 800 switches, a large part of them never being used as they would result in useless circuit setups. The suggested solution is considered a reasonable compromise between cost and flexibility.

\section{Analogue input buffers}

The connector on the far right side of the board in figure 1 is the connector for the data acquisition module. This is designed to allow an adapter to connect the motherboard to many different acquisition solutions - from the industry standard NI-DAQ series [9] to the simple, low cost micro controller based solutions like the PIC18 series [10] from Microchip and the different variants of Arduino [11]. Any custom made sampler will also be supported if so desired by the system designer. As was the case with the configuration block, this connector can also support long pins allowing for the use of wire wraps for temporary connections during prototyping, but it will also support standard flat-cable connectors or smaller acquisition boards mounted directly into the socket.

Analogue buffers for the analogue input signals are integrated in the motherboard. These can be seen as the components between the connector for the acquisition module and the configuration block. All low-cost multichannel analogue acquisition solutions are built using a single analogue to digital converter (ADC) with the inputs connected via an analogue multiplexer. This is done so in order to reduce the cost of implementing several relatively expensive ADCs. Separate ADCs are only found in more expensive, simultaneously sampling solutions when this is required.

The practical consequence of this is that input buffers are required between each of the high impedance analogue signal sources and their respective analogue input to the ADC. The need for the buffers comes as a result of the significant input capacitance of the sample-and-hold circuit placed after the analogue multiplexer in the ADC. Although the ADC has a high input resistance, giving an input bias current in the order of a few micro- or nano amperes, the dynamic characteristics of the analogue input causes the input impedance to low.

The result of this input capacitance is crosstalk between the input channels, meaning that if a signal source with low output resistance is connected to an input channel which is selected just before an input channel driven by a high output resistance source, the sensed signal of the latter will be highly influenced by the former signal. The solution to this problem is to simply set up buffers with high input resistance and low output resistance, where the simplest setup is the voltage follower. These buffers are mounted on the motherboard between the analogue input pins of the configuration block and the analogue inputs of the acquisition board connector.

\section{Power supplies}

For connecting an external power supply to the motherboard, the small connector at the bottom of the circuit board of figure 1 is used. Here it is found connections for $\pm 15 \mathrm{~V}$ or $\pm 12 \mathrm{~V}$, in addition to ground pins. A digital power supply is realized using a standard 7805 voltage regulator mounted on the motherboard. The integration of the digital supply on the motherboard is motivated by the analogue multiplexers requiring a higher voltage applied to the analogue positive supply terminal than the voltage applied to the digital supply terminal. If the digital supply is connected without the analogue supply, high currents will flow into the digital supply pin, possibly damaging the circuit. The voltage regulator on the motherboard is supplied from the positive analogue supply, making it impossible to have a larger digital supply voltage than the analogue supply voltage. It can be argued that this will introduce interference between the analogue and the digital power systems. This is however not considered a large problem for two reasons. First, the attenuation of noise components in the power systems is largely reduced by the filter capacitors which are mounted on the motherboard for reduction of EMI. Second, the digital communication, which is the main concern for interference transmitted from the digital to the analogue system, is only used during configuration of the component boards. When running the experiment, there is no activity in the digital part of the system, resulting in stationary voltages and currents exhibiting only DC-components. These are almost completely damped out between the two systems, making the interference problem insignificant.

In order to protect the circuit under test from possibly damaging over-currents, the current in both the positive and negative power supply should be monitored on the motherboard. If the current increases above a set limit, the power supply must be disconnected. This can be done by turning off a pair of transistors, but this system is not yet implemented in the solution presented. This would also require some sort of configuration of the current limits and means of reporting the over current state to the remote laboratory web server. For the current implementation, this is solved by designing the experiment circuit so that it is impossible to reach a state where over-currents, overvoltages or to high effects can develop in the components.

\section{E. Component boards}

Table 2 shows the pin assignment for the component boards. The pins named "analogue connect" are connected to the configuration block. The analogue voltage supply 
$(15 \mathrm{~V})$ is used for powering the switching network, but can also be used to power the remotely configurable components used in the experiments, such as operational amplifiers.

A typical circuit to be realised using the remote laboratory platform described in this paper is the small signal BJT amplifier shown in figure 2. When setting up a circuit for a remote laboratory experiment, a prerequisite is that the values or possibly type of the different components must be remotely controllable or selectable. For the circuit of figure 2 , this means that all the different resistors and capacitors must be configurable, i.e. their values must be possible to change. Replacing the transistor with transistors of different types would also be good. This is achieved by utilising the switching elements found on the component boards. The simple scheme is demonstrated by the component boards shown in figure 3 , with schematics shown in figure 4 through 6.

TABLE II.

PIN CONFIGURATION FOR THE COMPONENT BOARDS

\begin{tabular}{|l|c|c|l|}
\hline \multicolumn{4}{|c|}{ Motherboard pin assignment for the component board connectors } \\
\hline Signal & Pin\# & Pin\# & \multicolumn{1}{c|}{ Signal } \\
\hline +15V power supply & 1 & 2 & -15 V power supply \\
\hline Ground & 3 & 4 & +5 V digital supply \\
\hline Data 0 & 5 & 6 & Data 1 \\
\hline Data 2 & 7 & 8 & Data 3 \\
\hline Data 4 & 9 & 10 & Data 5 \\
\hline Data 6 & 11 & 12 & Data 7 \\
\hline Analogue connect 0 & 13 & 14 & Data Enable \\
\hline Analogue connect 2 & 15 & 16 & Analogue connect 1 \\
\hline
\end{tabular}

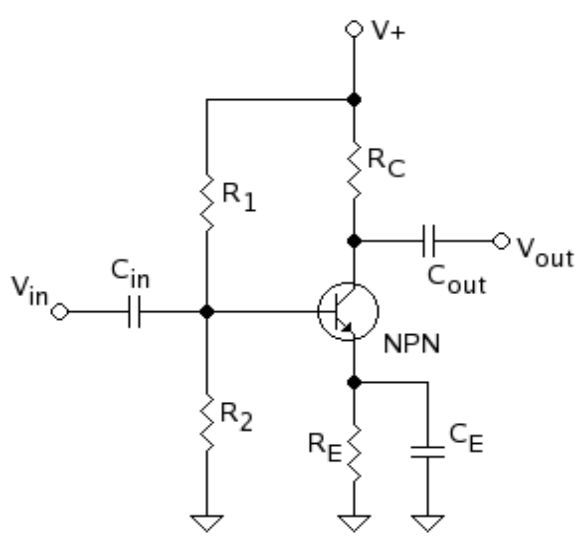

Figure 2. Schematic for a small signal common emitter BJT amplifier

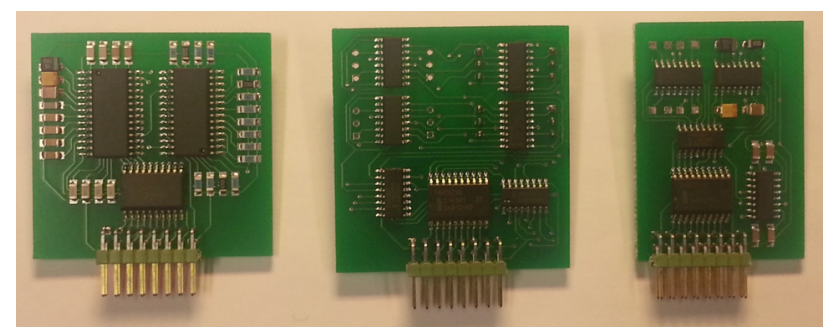

Figure 3. Component boards realising a voltage divider, one transistor and a single low-ohm resistor.
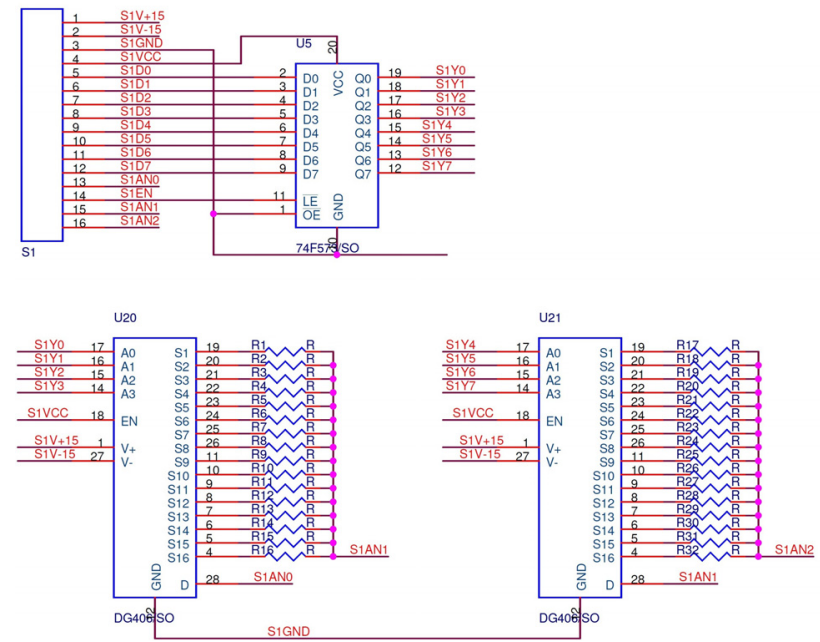

Figure 4. Schematic for a voltage divider circuit

This strategy is previously demonstrated in [12] where a remote laboratory for a small signal amplifier using a BJT is built using remote configurable resistors and capacitors. Digital buffer circuits ('573) are used to store the configuration data that is transferred via the data bus to all component boards. Separate enable signals for each board are used in order to reduce the complexity of the configuration protocol, and to avoid timing problems and glitches when using an address/data bus combination. Creating a more complex bus topology is possible, but would cause tremendous obstacles when implementing the control system in certain programming languages, such as the NI-DAQ in LabVIEW [13], when these are chosen as the programming solution.

The component realized in figure 3 and 4 is a voltage divider consisting of two resistors with 16 possible values each. The configuration data is fed directly to the switching elements, found as the analogue multiplexers U20 and U21: DG406 [14]. Here it can be seen that all possible resistance values are found as normal resistors that are switched in and out at the choice of the user. The resistors numbered R1 to R16 all have one terminal connected to a common net named "S1AN1" in the schematic, and the common point of their multiplexer is connected to the net named "S1AN0". The other half of the resistor pair is connected to nets "S1AN1" and "S1AN2", making the net "S1AN1" the midpoint of the voltage divider. These three nets are connected to the configuration block as described in the previous subsection, and constitute the three points of the remotely configurable voltage divider in the laboratory circuit. The analogue multiplexer used does exhibit an internal resistance of maximum $100 \Omega$, with a typical value of $50 \Omega$. The worst case error on resistance will then be for the lowest value of the resistor selectable. For the setup described in section III, this value will be $1.8 \mathrm{k} \Omega$, which gives equivalent to approximately $5 \%$ increase in the resistor value. When using $1 \%$ resistors in the component board, this gives a total of around $6 \%$ error. This error can be reduced by selecting resistors for the component boards from the E196 series that have slightly lower resistance than specified, so that the error is distributed more evenly around the desired value, taking into account the internal resistance of the multiplexers. Alternatively the users can be presented with slightly higher values in the web interface for the remote lab, but this is undesirable 
as it will present what will be perceived as non-standard values.

The voltage divider modules can also be used for the implementation of combined resistor/capacitor circuits, by simply replacing the resistors of one half of the pair with capacitors. This is utilised in the example experiment as shown in table III in the next section. This allows for very efficient re-use of different component boards, as this type of component combination are used in a large number of circuits, such as amplifiers, analogue filters - active or passive, oscillators, and other op-amp circuits.

Other parts of the circuit will either require lower resistances or have larger currents. In this case the analogue switches offer an internal resistance of less than $10 \Omega$. For the circuit presented in figure 2 , this is typically required for the emitter capacitor, and in the case of split emitter resistor, the resistor closest to the emitter point, where the peak currents reach a level where the internal resistance causes a significant voltage drop, if the analogue multiplexers are used. Using the analogue switches instead, can reduce this voltage drop to $1 / 10$, which is adequate for this type of application.

The transistor component board has 8 places for transistors. The reason for this high number is that this setup allows the user to put a combination of BJT and FET transistors on the same module board. This makes it possible to have the same setup for running both BJT amplifier laboratories as well as FET amplifier laborato-
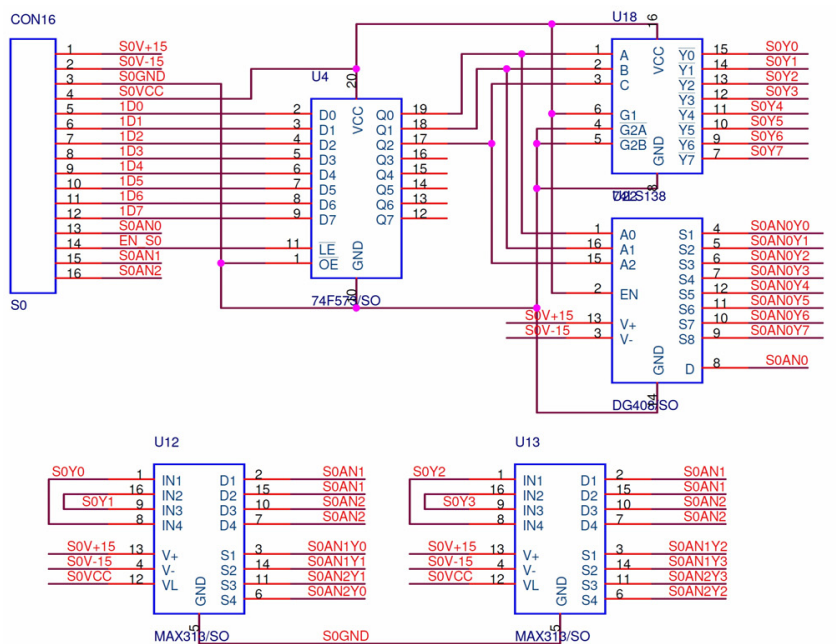

U15

$\mathrm{U} 16$
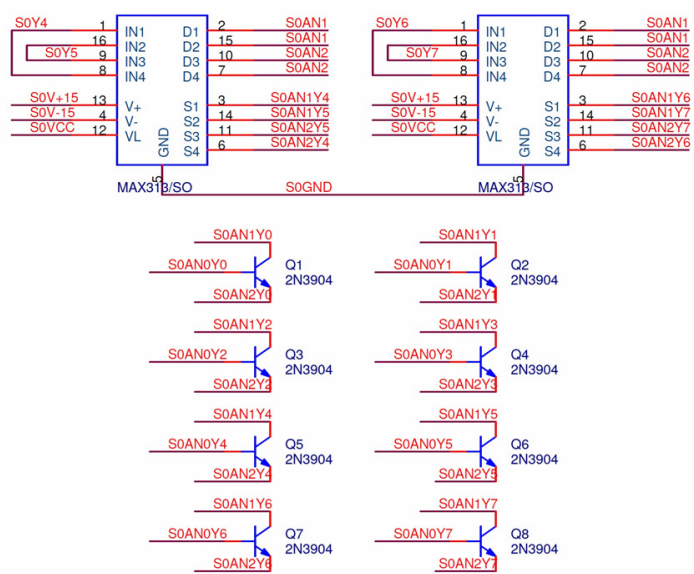

Figure 5. Schematic for selecting on of 8 different transistors (BJT or FET).
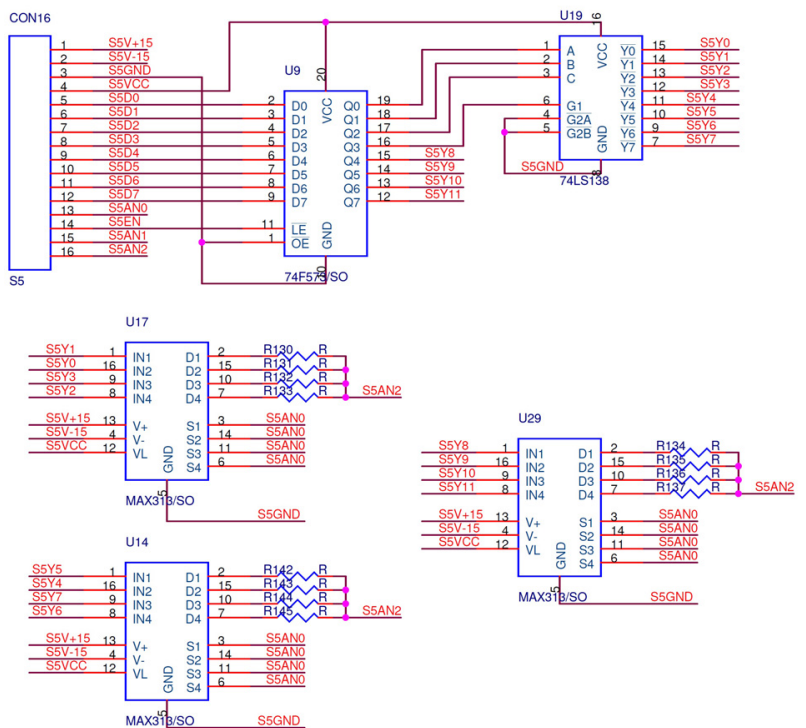

Figure 6. Schematic for a single low-ohm resistor circuit

ries. In addition, several different variants of a particular transistor can be used. An example of this can be found in the 2N3904, which comes in three different variants, A, B and $\mathrm{C}$, which all has different current amplification factors $(\beta D C$ and $\beta A C)$. This factor also varies with production, and a laboratory giving the user the chance of testing three different transistor of exactly the same type, allows for important demonstration of bias network stability, without time-consuming replacement of transistors on a breadboard. This type of demonstration is also not easily achievable when using simulation software.

\section{EXAMPLE OF A LABORATORY SETUP}

As an example if the use of the proposed system, a remote laboratory setup is now described. The laboratory setup is made for a remote lab with a standard small signal common emitter BJT amplifier circuit. A basic schematic of the circuit is shown in figure 2 .

In this setup 7 component boards are used, as shown in figure 7. Here it can be seen the component boards stacked in one row. The boards are listed in table III above. The component boards mounted in socket 7 through 10 have a lowest resistance much larger than that of the multiplexer $(<100 \mathrm{Ohms})$, and the normal analogue multiplexer is therefore used. There is also a desire to give large freedom in the number of different values to choose from, in order to allow the users to play with different output resistance of the voltage divider, relative to the base-emitter circuit.

The emitter capacitor allows for only 7 different values, in addition to open and short circuit, but this is considered enough to show the effect this has on the frequency response of the amplifier. The emitter resistance is divided in two parts, allowing the user to implement the "swamping" emitter coupling if desired. The number of selectable values for RE1 is limited to 12 due to the analogue switches being used. The switches are chosen as a result of the higher maximum peak current that might run through this part of the circuit. The same argument is used for the emitter capacitor and the output resistance. The other emitter resistor, RE2, use a normal multiplexer, as the current through this component will be limited due to 


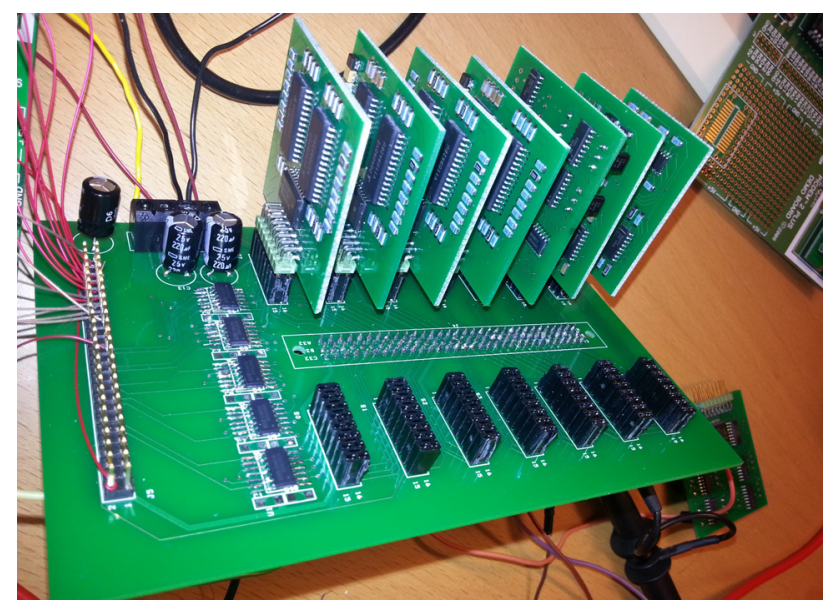

Figure 7. Setup of the hardware for the remote laboratory

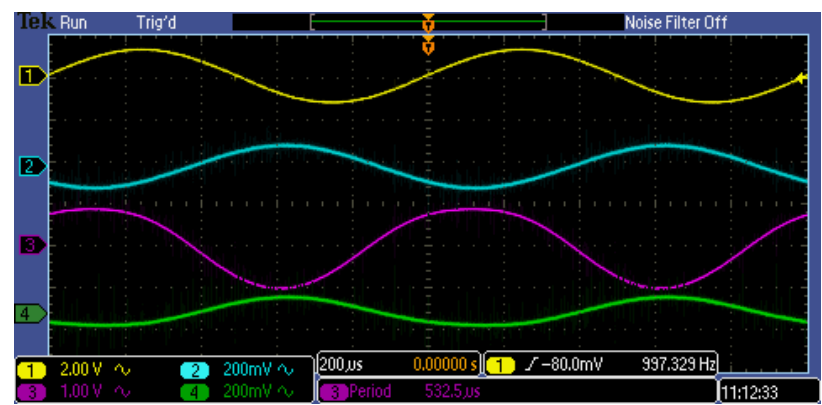

Figure 8. Experiment recording case \#1

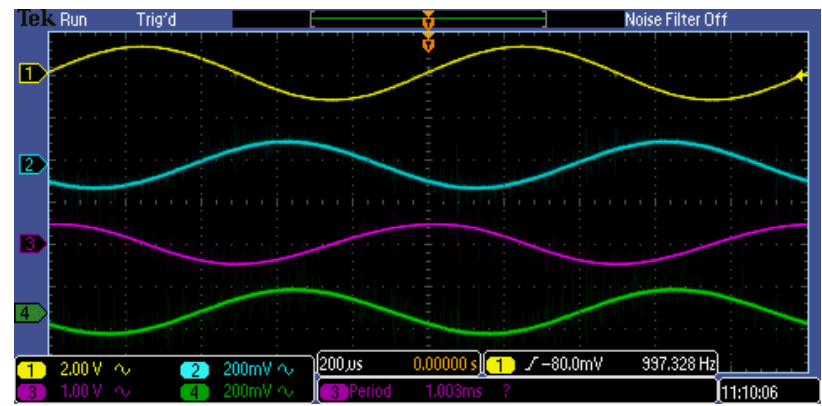

Figure 9. Experiment recording case \#2

the possible inclusion of the emitter capacitor. If the emitter capacitor is removed from the circuit, the current peak through this component will in any way not exceed levels where the voltage drop will be significant.

It is then possible to set up the circuit without the emitter capacitor for measuring the effect of no stabilizing element in this part of the circuit, and it is possible to select between 4 different transistors, possibly all with the same marking, but slightly different characteristics. This is done for the user to test the stability of the bias network selected, when the parameters of the transistor changes within the normal production range.

Figure 8 and 9 show two different experiment runs, using different values of the components. The input signal is the same in both cases. The signals are numbered 1 through 4 from top to bottom. Signal 1 is the input signal, 2 is the signal measured at the base of the transistor, 3 is the voltage at the emitter point, while 4 is the voltage at the output. The graphs show that it is possible to reconfigure the system using the data control signals, and that this actually change the behaviour of the circuit.
TABLE III.

PIN CONFIGURATION FOR THE DAUGHTERBOARDS

\begin{tabular}{|l|c|c|}
\hline \multicolumn{1}{|c|}{ Component } & Selectable values & Socket \\
\hline $\mathrm{C}_{\mathrm{IN}}$ and $\mathrm{R}_{\mathrm{S}}$ & $\begin{array}{c}\mathrm{C}_{\mathrm{IN}}: 0.1 \mu \mathrm{F}, 0.15 \mu \mathrm{F}, 0.22 \mu \mathrm{F}, 1 \mu \mathrm{F}, 2.2 \mu \mathrm{F}, \\
4.7 \mu \mathrm{F}, 10 \mu \mathrm{F} \\
\mathrm{R}_{\mathrm{S}}: \mathrm{E} 12 \text { series from } 1.8 \mathrm{k} \Omega \text { to } 33 \mathrm{k} \Omega .\end{array}$ & $\mathrm{S} 8$ \\
\hline $\mathrm{R}_{1}$ and $\mathrm{R}_{\mathrm{C}}$ & $\begin{array}{c}\text { Both: E12 series from } 1.8 \mathrm{k} \Omega \text { to } 33 \mathrm{k} \Omega . \\
\mathrm{R}_{2} \text { and } \mathrm{R}_{\mathrm{E} 2}\end{array}$ & $\mathrm{~S} 7$ \\
\hline $\mathrm{C}_{\mathrm{OUT}}$ and $\mathrm{R}_{\mathrm{L}}$ & $\begin{array}{c}\text { Both: } \mathrm{C} 12 \text { series from } 1.8 \mathrm{k} \Omega \text { to } 33 \mathrm{k} \Omega . \\
2.2 \mu \mathrm{F}, 4.7 \mu \mathrm{F}, 10 \mu \mathrm{F}\end{array}$ & $\mathrm{S} 9$ \\
\hline $\mathrm{R}_{\mathrm{E} 1}$ & $\mathrm{R}_{\mathrm{L}}: \mathrm{E} 12$ series from $1.8 \mathrm{k} \Omega$ to $33 \mathrm{k} \Omega$. & $\mathrm{S} 10$ \\
\hline $\mathrm{C}_{\mathrm{E}}$ & $\begin{array}{c}\mathrm{E} 6 \text { series from } 68 \Omega \text { to } 3.3 \mathrm{k} \Omega . \\
\mathrm{C}: \text { Open, short circuit, } 0.1 \mu \mathrm{F}, 0.15 \mu \mathrm{F}, \\
0.22 \mu \mathrm{F}, 1 \mu \mathrm{F}, 2.2 \mu \mathrm{F}, 4.7 \mu \mathrm{F}, 10 \mu \mathrm{F}\end{array}$ & $\mathrm{S} 13$ \\
\hline BJT & 8 types of $2 \mathrm{~N} 3904$ selectable & $\mathrm{S} 12$ \\
\hline
\end{tabular}

\section{CONCLUSIONS}

In this paper it is demonstrated newly designed standardized remote laboratory platform from which to make development of new laboratories easier and faster, but still at a low cost. The solution facilitates in a natural way the development of remote laboratories through the prototyping phase through to a robust permanent installation. The platform is not tied to a specific data acquisition solution but rather designed to make it possible to easily change from one data acquisition solution to another.

\section{REFERENCES}

[1] S. Ursulet and D. Gillet, "Introducing flexibility in traditional engineering education by providing dedicated on-line experimentation and tutoring resources," presented at the The International Conference on Engineering Education, Manchester, UK, 2002

[2] O. H. Graven and D. Samuelsen, "Building and configuring a power supply in a remote lab experiment," presented at the AsiaMIC Phuket, Thailand, 2010.

[3] D. Samuelsen and O. H. Graven, "Using a remote lab for real physical measurements on an electric motor," presented at the AsiaMIC, Phuket, Thailand, 2010.

[4] B. Pradarelli, L. Latorre, and P. Nouet, "Integrated circuits testing: Remote access to test equipment for labs and engineering," iJOE, vol. 5, Special Issue 1: REV2009, 2009.

[5] Faculdade de Engenharia da Universidade do Porto, "eLabs FEUP," http://elabs.fe.up.pt/ , 2009.

[6] Massachusetts Institute of Technology, "iLabs: Internet access to real labs," http://icampus.mit.edu/iLabs/, 2009.

[7] C. Maier and M. Niederstätter, "Lab2go: A Repository to Locate Online Laboratories," International Journal of Online Engineering (iJOE), vol. 6, pp. 12-17, 2010.

[8] J. M. M. Ferreira and D. Mueller, "Online labs and the MARVEL experience," International Journal of Online Engineering vol. 1, 2005.

[9] NI Multifinction Data Aquisition. [Web page] [cited 2012.04.04]; Available from: http://www.ni.com/dataacquisition/multifunction/.

[10] Microchip 8-bit Micro controllers. [Web page] [cited 2012.04.04] Available: http://www.microchip.com/pagehandler/en_us/family/ $\underline{8 \text { bit/. }}$

[11] Arduino-ArduinoBoardMega, [Web page] [cited 2012.04.04] Available from: http://arduino.cc/en/Main/ArduinoBoardMega.

[12] Samuelsen, D.A.H.; Graven, O.H.; , "Remote lab for experiments on a small-signal bipolar-junction transistor amplifier," Global Engineering Education Conference (EDUCON), 2011 IEEE , vol., no., pp.721-725, 4-6 April 2011 http://dx.doi.org/10.1109/ EDUCON.2011.5773218 
[13] National Instruments, National Instruments, LabVIEW, [Web page] [cited 2012.04.04] Available from: http://www.ni.com/ labview/

[14] Intersil, Intersil, Datasheet FN3116.9: "DG406, DG407”, [Web page] [cited 2012.04.04] Available from: http://www.intersil.com/ data/fn/fn3116.pdf

\section{AUTHORS}

D. A. H. Samuelsen is with Buskerud University College, Department for Technology, Kongsberg, Norway (email: dag.samuelsen@hibu.no).
O. H. Graven is with Buskerud University College, Department for Technology, Kongsberg, Norway (e-mail: olaf.hallan.graven@hibu.no).

This article is an extended and modified version of a paper presented at the International Conference on Remote Engineering \& Virtual Instrumentation (REV2012), held at University of Deusto, Bilbao, Spain, July 4-6, 2012Received 25 February 2013. Published as resubmitted by the authors 20 March 2013. 